\title{
Molecular differentiation and specialization of vascular beds
}

\author{
Susana F. Rocha $\cdot$ Ralf H. Adams
}

Received: 18 January 2009/Accepted: 22 January 2009/Published online: 12 February 2009

(C) The Author(s) 2009. This article is published with open access at Springerlink.com

\begin{abstract}
Transport in the large and complex bodies of vertebrate organisms is mediated by extensive and highly branched tubular networks that are formed by endothelial cells. Blood vessels are responsible for systemic circulation, while the lymphatic vasculature drains extravasated plasma, proteins, particles, and cells from the interstitium. Endothelial cells of blood vessels and lymphatic vessels can be distinguished by the expression of certain molecular markers, which accompany or even contribute to functional and morphological differences. Even within the blood vessel network, some molecules and pathways selectively mark the endothelium of arteries, veins and capillaries and are thought to contribute to the differentiation of these vessels. Moreover, microvessels can acquire organ-specific specialization in response to local tissue-derived signals. This review summarizes molecular markers and pathways that are specifically expressed in the endothelium of certain vascular beds and vessel types. Special attention will be given to known functional roles in the morphogenesis of these vessels.
\end{abstract}

Keywords Artery · Vein - Development - Endothelium . Notch · VEGF · Blood-brain-barrier · Lymphatic . Prox 1

S. F. Rocha $\cdot$ R. H. Adams $(\bowtie)$

Department Tissue Morphogenesis, Max-Planck-Institute

for Molecular Biomedicine, University of Münster,

48149 Munster, Germany

e-mail: ralf.adams@mpi-muenster.mpg.de

R. H. Adams

Faculty of Medicine, University of Münster,

Munster, Germany

\section{Introduction}

Until the late 90s, it was thought that the characteristic features of arteries and veins were controlled by hemodynamic forces, such as blood pressure and differences in oxygenation. This idea was first challenged about a decade ago with the description of the differential expression of ephrin-B2 and of its receptor, EphB4, in embryonic arterial and venous cells, respectively, even before the onset of blood-flow and heart beat [1-3]. Since then, additional arterial-venous (AV) markers have been identified (Fig. 1). For instance, arterial cells are known to specifically express the gap junction proteins Connexin-37 (Cx37) and Connexin-40 (Cx40) [4-6], components of the Notch pathway, such as Dll4 [7-9], as well as the VEGF co-receptor Neuropilin-1 (NRP1) [10-12]. On the other hand, venous cells specifically (or at least predominantly) express certain members of the VEGF pathway, such as the co-receptor Neuropilin-2 (NRP2) [10, 13, 14] and receptor VEGFR3 [15-17], COUP-TFII that negatively regulates the Notch pathway [18], and the Apj receptor [19]. Nevertheless, it is not fully understood to what extent some of these AV markers are essential for the determination of the endothelial cell (EC) fate. Indicating multiple functional roles in the vasculature, several of these molecules are also expressed in growing capillary beds and control endothelial sprouting angiogenesis in addition to $\mathrm{AV}$ differentiation (Fig. 1). It also remains to be resolved, to which extent genetics and hemodynamic forces may act in an integrated, interdependent fashion. For example, it has been shown that shear forces derived from blood flow induce the expression of transcription factors, such as Krüppel-like factor 2 (Klf2) and of its putative downstream targets [2022], thereby establishing a link between hemodynamic and genetic regulation. 
Fig. 1 Vessel-type specific markers. Expression of selected molecular markers in the endothelium of an artery (red), vein (blue) and lymphatic vessels (green). While some markers are expressed in a strictly AV-specific fashion, others extend into the capillary network and have been linked to angiogenic growth and sprouting. VSMCs on blood vessels (red/blue) and valvecontaining lymphatic collecting ducts as well as pericytes covering capillaries (yellow) are indicated

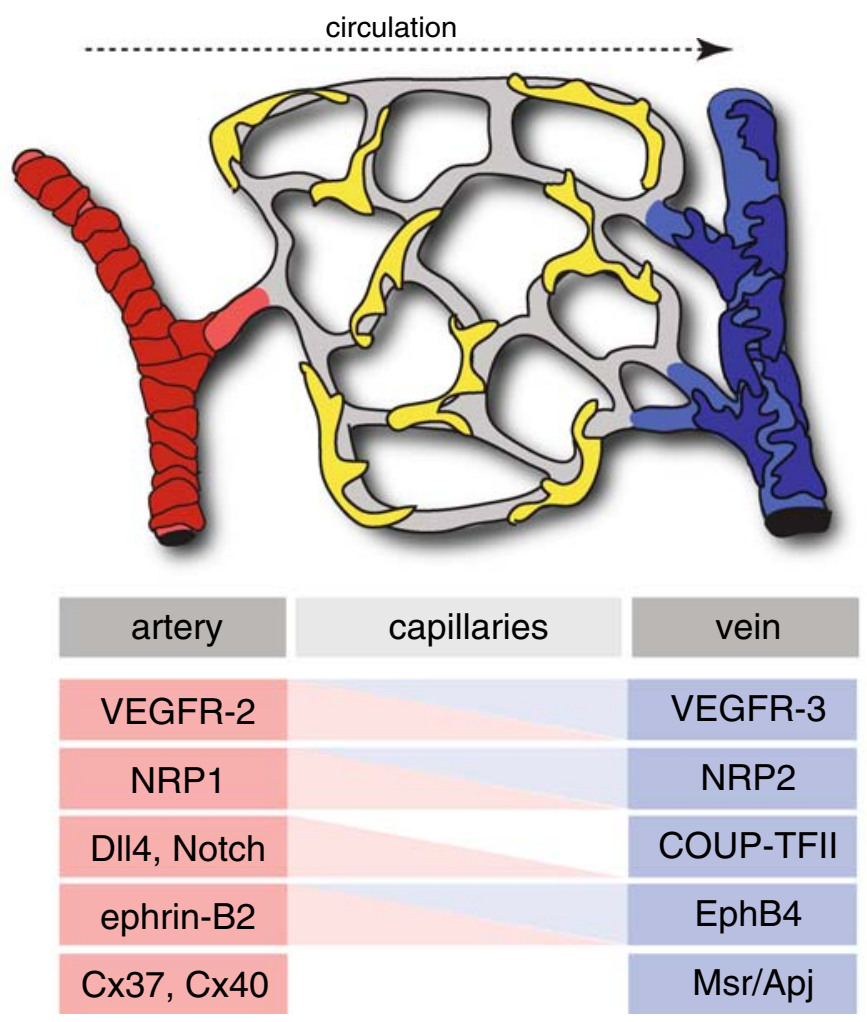

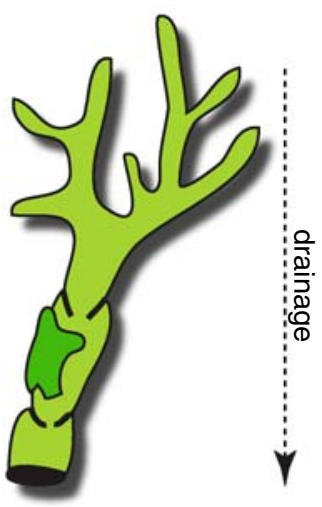

lymphatics

VEGFR-3, NRP2

LYVE-1, podoplanin

Prox1

ephrin-B2/EphB4

CCL21

\section{Cellular processes contribution to AV differentiation}

While it has been proposed that ECs committed to the arterial or venous fate are already molecularly distinct in the primitive vascular plexus of the yolk sac [1], arteries and veins are formed from capillary ECs without apparent predetermined AV commitment in many other tissues (Fig. 2). Here, arterial-venous differentiation involves the remodeling of primitive capillary beds into a hierarchial network of arteries, capillaries, and veins with distinct morphologies and gene expression profiles. This also indicates that capillary ECs possess a significant amount of plasticity and can differentiate into venous or arterial endothelial cells depending on their location within a remodeling vascular bed. The detailed cellular and molecular processes that are part of the AV remodeling program are incompletely understood. However, since arteries have few side branches, their differentiation from a dense capillary network has to involve extensive pruning processes. Indeed, retraction figures, i.e., local thinning and detachment of endothelial connections, are abundant during early as well as later stages of arterial morphogenesis [23] (Fig. 2). Signs of pruning can be also seen around veins and even in capillary beds but are less frequent when compared with the peri-arterial space. As a consequence, one characteristic of arteries is that they are surrounded by an almost completely avascular (i.e., capillary-free) zone, which increases with arterial caliber and probably indicates sufficient oxygenation of the peri-arterial tissue [23] (Fig. 2). Another typical feature of arteries is their slender and straight morphology (Fig. 2), which is thought to be an adaptation to high flow speeds and local shear stress [24, 25]. Conversely, developing veins appear more irregular and have a larger diameter, features which are also seen as a consequence of local hemodynamic properties. At the same time, EC proliferation in capillaries and veins is high when compared with the arterial endothelium, which may, together with cell shape changes (primarily EC elongation/stretching and parallel alignment), contribute to the characteristic morphologies of developing arteries and veins.

Differential recruitment and association of mural cells is a further feature of the AV differentiation cascade [26-28]. Vascular smooth muscle cell (VSMC) coverage is more pronounced on arteries and circumferential alignment of these cells (perpendicular to the direction of blood flow) is likely to maximize structural support given to the endothelium [29-31]. Certain smooth muscle differentiation markers, like the protein smoothelin which also directly contributes to contractility, are expressed in VSMCs of perinatal arteries but not in veins $[32,33]$. Venous smooth muscle cells are also less abundant and their alignment is limited. Likewise, certain matrix proteins and elastic fibers are predominantly or exclusively found in the arterial vessel wall [34-36]. The factors controlling these distinct 


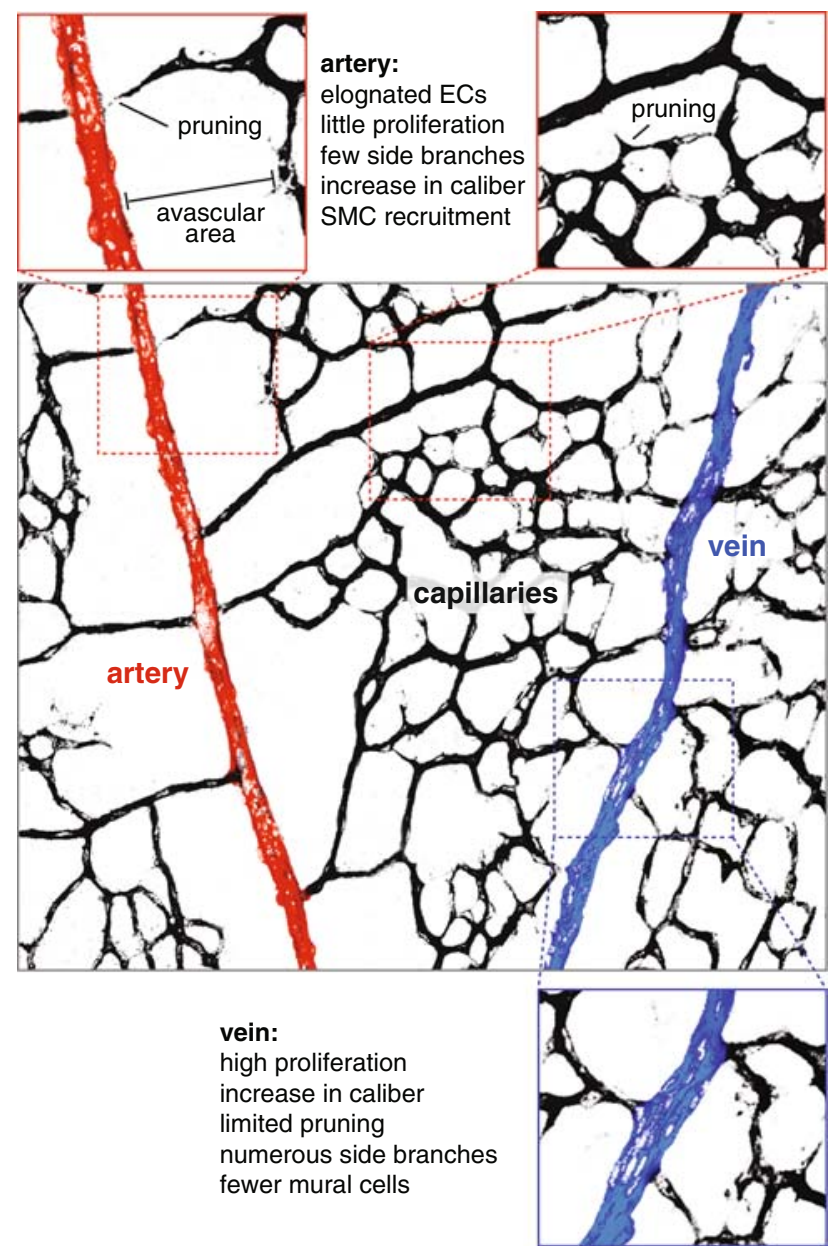

Fig. 2 Processes during AV remodeling. Schematic representation (based on a real isolectin B4 staining of retinal blood vessels) of cellular processes in growing vascular beds. Higher magnifications of insets show pruning processes during arterial development (red boxes) and features of the venous circulation (blue)

recruitment and differentiation features remain to be identified, but known regulators of mural cell recruitment, such as platelet-derived growth factor B (PDGF-B) and its receptor PDGFR $\beta$ [26, 37], TGF- $\beta$ and its receptors [38-42], angiopoietin-Tie2 [43-45], and ephrin-B2 [46, 47], are likely candidates. Likewise, vessel type-specific expression of Klf2, which is induced in response to blood flowderived shear forces, regulates VSMC migration as well as vessel wall assembly, and thereby integrates hemodynamic and genetic aspects of arterial differentiation [20-22, 48].

\section{The many roles of VEGF signaling}

The VEGF pathway is critical for blood vessel formation, given its participation in a variety of vascular processes including EC proliferation, migration, survival, and arterial-venous cell fate specification $[49,50]$. In mammals there are several VEGF ligands (VEGF-A, B, C, D and E) and receptors (VEGFR-1 to 3) that display distinct binding affinities enabling VEGF signaling to yield distinct signaling outputs and biological responses. VEGF signaling is required for proper arterial-venous specification and, accordingly, compromised VEGF (vegfaa) expression leads to an arterial-to-venous cell fate switch in zebrafish embryo [51]. While this first study focused on the role of VEGF signaling in the induction of the arterial cell fate, recent work has shown that the pathway is also required for the inhibition of the arterial fate in cells committed to venous differentiation [52]. How is VEGF signaling able to act on both arterial and venous endothelial cells and trigger distinct responses in each cell type?

The VEGF receptors show overlapping but distinct expression patterns. VEGFR2 is expressed in most or all endothelial cells, whereas VEGFR3 expression seems to be excluded from arterial cells [16, 17]. Moreover, the VEGF ligands display different binding affinities to each of the receptors. VEGF-A is only able to bind receptors VEGFR1 and 2, while VEGF-C and D have higher affinities for VEGFR-3. Additionally, it is known that VEGF-A-mediated signaling is more efficient in activating phospholipase $\mathrm{C}$-gamma (PKC- $\gamma$ ) and its downstream signaling partner, the mitogen-activated protein kinases (MAPKs) Erk1 and 2. In contrast, VEGF-D induces a strong activation of the protein kinase Akt through phosphatidylinositol 3-kinase (PI3K) [53]. The ligand VEGF-C is a potent activator of both MAPK and Akt [54].

Recent studies in zebrafish have shown that these two downstream targets of VEGF signaling, PKC- $\gamma /$ ERK and $\mathrm{PI} 3 \mathrm{~K} / \mathrm{Akt}$, have distinct roles in arterial $[55,56]$ and venous differentiation [52]. PLC- $\gamma 1 /$ Erk signaling induces proliferation and specification of arterial endothelial cells [55, 57], while PI3-K/Akt signaling inhibits PKC- $\gamma / \mathrm{MAPK}$ activation in cell culture and in zebrafish and thereby blocks the acquisition of the arterial cell fate [52]. Thus, the VEGF pathway participates in arterial-venous specification by differentially regulating the activation of downstream targets in arterial and venous cells.

Despite the striking evidence for distinct functional roles of VEGF-triggered signaling, it is still difficult to understand how certain downstream signal transduction cascades get selectively activated in response to VEGF-VEGFR interactions. Thus, it is likely that additional players, such as Neuropilin family (NRP) molecules, can further modulate the final output of VEGF signaling. NRP1 and NRP2 are transmembrane glycoproteins that function as nonsignal transducing co-receptors of VEGF. NRP1 forms a co-receptor complex with VEGFR-2 and thereby enhances the binding affinity of VEGFR-2 to a specific isoform of VEGF-A (VEGF164), which plays a pivotal role in vascular development. NRP1-deficient mice are embryonically 
lethal at midgestation due to defects in vascular remodeling of the yolk sac and impairment in the development of aortic arches and large vessels [58]. EC-specific deletion of NRP1 leads to the loss of specific arterial markers, such as ephrin-B2 and Connexin40 [59]. Knockout mice lacking NRP2 are viable and display axon guidance defects, but not any overt blood vessel phenotype [60, 61]. However, the formation of the lymphatic vessels is impaired in these mutants [14]. NRP2 expression in venous and lymphatic ECs mimics that of VEGFR-3. Indeed, VEGF-C and D have been shown to bind to NRP2, which enhances VEGF receptor signaling in cultured cells and lymphangiogenesis in tumors [62-64].

\section{Notch signaling and arterial-venous specification}

Notch signaling is an evolutionarily conserved pathway that is involved in a wide range of biological processes including cell fate determination, proliferation, and survival $[65,66]$. Signaling through the Notch pathway occurs through direct cell-cell communication and is mediated by contactdependent receptor and ligand interactions. In mammals, there are four distinct Notch receptors (Notch1-4) and five ligands that belong to the Delta (Delta-like 1, 3 and 4) or Jagged/Serrate protein families (Jag1 and Jag2). The analysis of Notch mutants has shown that the pathway is required for cell fate decisions, a well-known role in other systems such as the nervous system. Likewise for VEGF loss-of-function mutants [51], compromised Notch activity, both in mouse and zebrafish, has uncovered an important role in establishing the arterial cell fate [17, 67-70]. In addition, the Notch pathway also regulates sprouting angiogenesis through the 'selection' of tips cells at the angiogenic front [71-75]. The similarity between the phenotypes observed in mutants of the VEGF and Notch pathway suggests tightly controlled, interdependent regulatory relationships. Notch has been shown to act downstream of VEGF, since ectopic activation of Notch in a VEGF mutant background is sufficient to rescue vascular defects in zebrafish embryos [51]. Specifically, the Dll4 ligand, which is expressed exclusively in arterial ECs and angiogenic capillary beds, and the Notch1 receptor have been identified as downstream targets of VEGF signaling $[75,76]$. On the other hand, downregulation of VEGFR-2 and VEGFR-3 expression and upregulation of the antagonistically acting receptor VEGFR-1 are critical downstream responses to Notch activation in ECs [16, 71, 72, 74].

Recent in vitro work has shown that VEGF-mediated regulation of Notch occurs through transcription factors of the forkhead family, Foxc1 and Foxc2, which bind to the promoters of Dll4 and Hey2. The latter is a direct downstream target of Notch signaling in the vasculature and is involved in the regulation of gene transcription in response to Notch activation [77, 78]. Although the expression of Foxc1 and Foxc2 is not restricted to arterial ECs [77], it has been shown that the activity of these transcription factors is augmented by VEGF-activated Erk signaling in cultured cells. In contrast, PI3K signaling inhibits Foxc-mediated activation of the D114 and Hey2 promoters [78]. However, these results seem to be at odds with the higher levels of PLC- $\gamma 1 /$ Erk signaling reported for the zebrafish aorta [52], which should block the activity of Foxc transcription factors, and suggests that additional modes of regulation may exist.

The activity of Foxc1/c2, VEGF and Notch are known to promote the expression of arterial markers such as ephrin-B2 and Cx40, whereas the orphan nuclear receptor COUP-TFII (also known as NR2F2) has been identified as the first positive regulator of venous endothelial identity [18]. While COUP-TFII is present in both ECs and mural cells, endothelial expression is restricted to veins and excluded from arteries. COUP-TFII knockout embryos are lethal at day 12 after fertilization and exhibit a phenotype opposite to that observed upon loss of Notch activity: a partial loss of the venous cell fate indicated by reduced (but not completely absent) EphB4 expression, together with the ectopic expression of arterial markers, such as Dll4 and ephrin-B2 [18]. Ectopic expression of COUP-TFII in transgenic mice can suppress the expression of arterial markers in the dorsal aorta [18]. Although the mechanism through which COUP-TFII acts is not completely understood, its negative effect on D114 and NRP1 expression is likely to locally alter Notch as well VEGF signaling responses during the regulation of $\mathrm{AV}$ differentiation.

\section{Tissue-specific specialization of blood vessels}

The example of veins and arteries shows that ECs can differentiate into specialized subpopulations with characteristic gene expression profiles. Specific morphological and functional features of blood vessels in certain organs suggest the existence of an even larger degree of heterogeneity among ECs in terms of gene expression and signaling pathways. For example, the endothelium of endocrine glands, pancreas, intestine, kidney glomeruli and liver sinusoids contain small (60-70 nm in diameter) but densely clustered pore-like openings termed fenestra, which increase local permeability and are thought to facilitate the exchange between the circulation and the surrounding tissue. Some endothelial fenestrations contain a diaphragm, a central structure with wheel spoke-like extensions that subdivide the pore into several smaller openings. The type II membrane glycoprotein PV-1 (Plasmalemmal vesicle associated protein-1) is a molecular component of diaphragms, and it is both necessary 
and sufficient for diaphragm formation in cultured ECs [79, 80]. Anti-PV-1 immunofluorescence marks blood vessels containing diaphragmed fenestra, but is also present in some non-fenestrated vascular beds. Conversely, PV-1 is absent from certain fenestrated blood vessels (such as liver sinusoids) presumably because they lack diaphragms [81, 82].

Although very little is known about the formation of fenestra, it has been shown that their formation can be induced by VEGF-A [83-85] as well as endocrine gland vascular endothelial growth factor (EG-VEGF), a specialized and tissue-specific angiogenic molecule [86]. Further linking endothelial fenestrations to VEGF signaling, inhibition of VEGF activity in mice leads to the reduction of fenestrations and, concomitantly, the partial regression of capillaries in tissues containing fenestrated vascular beds [87-90]. This role of VEGF may also explain the appearance of ectopic endothelial fenestrations in the vasculature of tumors and in other pro-angiogenic disease settings [89, 91-93].

While endothelial fenestrations are associated with high permeability, the endothelium of the central nervous system forms a barrier (termed the blood-brain barrier, BBB) that tightly controls trans-endothelial transport and cell migration [94]. Continuous strands of tight junctions (TJs) containing claudin and occluding family membrane-spanning proteins seal brain ECs and thereby strictly limit the paracellular transport route. Enabling necessary transport across the BBB, ECs express specific transporters for glucose, amino acids, and other substances. The important role of the blood-brain barrier is emphasized by its breakdown in human diseases. For example, immune cells gain access to the normally immunoprivileged brain tissue and trigger harmful inflammatory processes in the autoimmune disease multiple sclerosis [94].

Recent work has established that the formation of the BBB during development is controlled by canonical Wnt signaling $[95,96]$. Wnt7a/b double mutants or embryos lacking endothelial expression of $\beta$-catenin display extensive hemorrhaging and reduced vascularization of the CNS.
The glucose transporter GLUT-1, a BBB marker, is downregulated in these mutant embryos, whereas ectopic expression of Wnt7a is sufficient to induce GLUT-1-positive ECs outside the CNS [96]. A second and independent study that focused on postnatal formation and maturation of the BBB confirmed that endothelial $\beta$-catenin promotes Claudin-3 (Cldn3) expression and suppresses expression of PV-1 (in this study termed Pvlap), which is low/absent in the normal BBB endothelium but gets ectopically upregulated in mutant mice [95]. Furthermore, the authors show that Wnt3a can upregulate $C l d n 3$ levels in cultured ECs in a $\beta$-catenindependent fashion. Both studies imply canonical Wnt signaling and multiple Wnt genes in the formation of the BBB. Future work will have to address if Wnt signaling is also required for the maintenance of the $\mathrm{BBB}$ and whether activation of the pathway might be an approach to restore compromised barrier function in disease settings.

\section{Regulation of lymphatic endothelial cell identity}

Despite the fact that lymphatic ECs in the mouse are derived from venous ECs during embryonic development [97], the lymphatic endothelium expresses a set of specific genes that are not found on blood vessels (Fig. 1) [98, 99]. When morphogenesis of the lymphatic vasculature is initiated, differentiation of the first lymphatic ECs (LECs) in the cardinal vein can be detected by the expression of the homeobox-containing transcription factor Prox1 (prosperorelated homeobox gene 1) (Fig. 3). Prox1 is the master regulator of LEC differentiation and induces the expression of a battery of LEC-specific genes, while other genes, which are characteristic for the blood vessel endothelium, get suppressed [98-100]. In mice lacking Proxl, LEC differentiation fails and no lymphatic vasculature is formed [100].

Recently, it has been shown that the role of Prox1 is not confined to lymphatic development in the embryo, but actually extends into adulthood. Tamoxifen-inducible Cre-mediated (i.e., temporally controlled) inactivation of
Fig. 3 Induction of the lymphatic vasculature. Expression of Sox 18 in selected venous ECs triggers the expression of the lymphatic master regulator Prox1. Prox 1 induces expression of other markers and regulators of the lymphatic vasculature such as VEGFR-3, which controls LEC proliferation, migration and numerous other aspects of lymphangiogenic growth

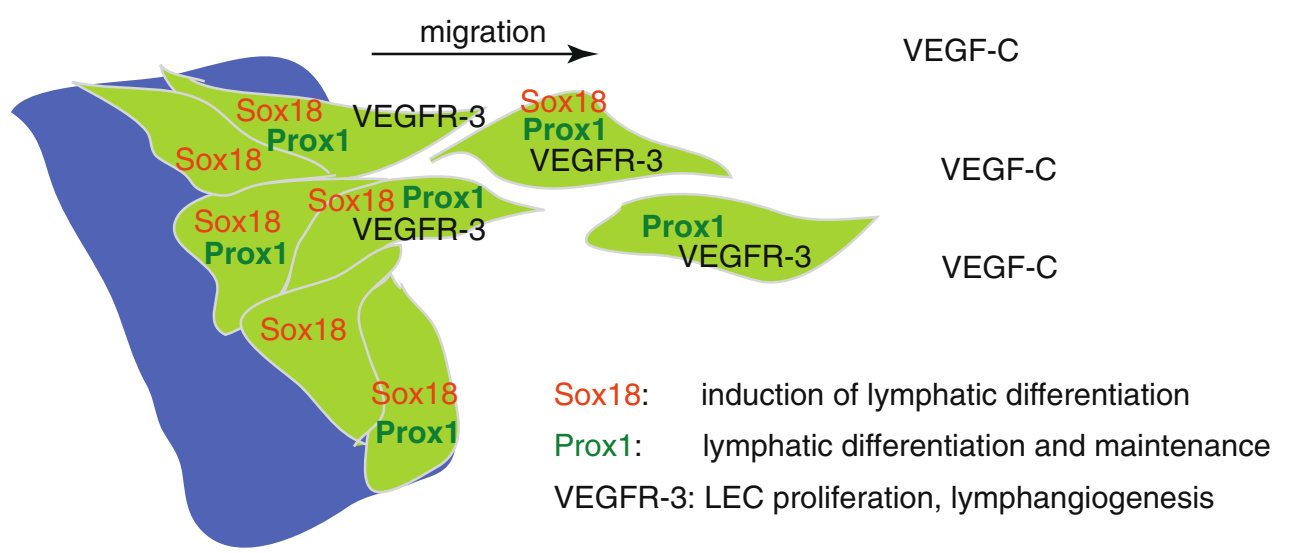


the Prox 1 gene at various embryonic or postnatal stages leads to lymphatic vessel defects, prominent edema and the downregulation of LEC markers like podoplanin, CCL21/SLC and LYVE-1 [101]. At the same time, endoglin or CD34, markers characteristic for blood vessel ECs (BECs), are upregulated and mutant lymphatic capillaries (initial lymphatics) acquire an ectopic coverage by $\alpha$-smooth muscle actin-positive perivascular cells reminiscent of the mural coverage of blood vessels [101]. The surprising conclusion that continued Prox 1 expression is required to maintain LEC differentiation raises the question whether lost expression or dysfunction of Prox-1 might be linked to human pathologies, particularly to settings where lymphatic vessels are compromised or ECs lack a clear BEC/LEC identity [102-104].

Another important task will be the identification of factors required for the induction or maintenance of Prox 1 expression. In the early embryo, one such upstream regulator is the transcription factor Sox18 [105] (Fig. 3). Dominant negative mutations of the Sox 18 gene in naturally occurring mouse mutants of the ragged allelic series cause chylous ascites and edema. Likewise, dysfunction of the Sox18 gene is linked to the hypotrichosis-lymphedema-telangiectasia syndrome in humans [106]. While homozygous Sox18-deficient mice in a mixed 129/CD1 background do not display vessel defects, perhaps due to genetic compensation by the related Sox family members Sox 7 and Sox 17, knockout mice in a purebred C57/B16 background have been recently found to develop lethal fetal edema [105]. Like Prox1, Sox 18 is also expressed in an EC cluster within the cardinal vein and even precedes the onset of Prox 1 expression by a whole day. Explaining the lymphatic defects seen in Sox18 mice, no induction of Prox1-positive ECs occurs in the cardinal vein of these mutants. Conversely, forced expression of Sox18 in culture differentiating, embryonic stem cell-derived ECs induces the upregulation of Prox 1 and other lymphatic signature genes. Indicating that Sox 18 is a direct regulator of Proxl transcription, two Sox 18 binding sites in a proximal $4.1 \mathrm{~kb}$ Prox-1 promoter fragment are necessary for Prox-1 expression in vitro and in vivo [105].

Despite the important role of Sox 18 for the specification of the first LECs, the absence of vascular defects in Sox18deficient mutants in the mixed 129/CD1 background and expression of the gene in a fraction of embryonic blood vessels argue against a mandatory and general role of Sox 18 as an inducer of Prox 1 expression and therefore additional upstream regulators are likely to exist.

\section{Perspectives}

The examples above show how recent progress has provided us with an increasingly complex picture of the vasculature. Far from forming simple, blood-transporting tubes that are shaped by flow, ECs undergo a series of differentiation steps in response to intrinsic genetic programs as well as local tissue-derived signals. Future work will identify further markers that allow us to distinguish different vascular beds and vessel types. As the examples of Prox 1 and the Notch pathway show, some of these molecules might be even key regulators of EC differentiation processes. Unraveling of the genetic heterogeneity of signaling pathways in different vascular beds, vessels or even individual ECs will further improve our understanding of how the morphogenesis of the vasculature and organ-specific specialization processes are regulated. These findings may well help to explain changes in human pathologies and offer vital clues for the development of therapeutics. The large degree of plasticity of endothelial cells, which allows growth on demand after long periods of quiescence as well as differentiation/de-differentiation processes, suggests a significant potential for therapeutic interference even in the fully developed, adult organism.

Acknowledgments The authors thank the Max-Planck-Society and the University of Muenster for their support.

Open Access This article is distributed under the terms of the Creative Commons Attribution Noncommercial License which permits any noncommercial use, distribution, and reproduction in any medium, provided the original author(s) and source are credited.

\section{References}

1. Wang HU et al (1998) Molecular distinction and angiogenic interaction between embryonic arteries and veins revealed by ephrin-B2 and its receptor Eph-B4. Cell 93:741-753. doi:10.1016/ S0092-8674(00)81436-1

2. Gerety SS et al (1999) Symmetrical mutant phenotypes of the receptor EphB4 and its specific transmembrane ligand ephrin-B2 in cardiovascular development. Mol Cell 4:403-414. doi:10.1016/ S1097-2765(00)80342-1

3. Adams RH et al (1999) Roles of ephrinB ligands and EphB receptors in cardiovascular development: demarcation of arterial/venous domains, vascular morphogenesis, and sprouting angiogenesis. Genes Dev 13:295-306. doi:10.1101/gad.13.3. 295

4. Bastide B et al (1993) Gap junction protein connexin40 is preferentially expressed in vascular endothelium and conductive bundles of rat myocardium and is increased under hypertensive conditions. Circ Res 73:1138-1149

5. Reed KE et al (1993) Molecular cloning and functional expression of human connexin37, an endothelial cell gap junction protein. $\mathrm{J}$ Clin Invest 91:997-1004. doi:10.1172/JCI116321

6. Bruzzone R et al (1993) Connexin40, a component of gap junctions in vascular endothelium, is restricted in its ability to interact with other connexins. Mol Biol Cell 4:7-20

7. Shutter JR et al (2000) Dll4, a novel Notch ligand expressed in arterial endothelium. Genes Dev 14:1313-1318

8. Mailhos C et al (2001) Delta4, an endothelial specific notch ligand expressed at sites of physiological and tumor angiogenesis. 
Differentiation 69:135-144. doi:10.1046/j.1432-0436.2001.690 207.x

9. Claxton S, Fruttiger M (2004) Periodic Delta-like 4 expression in developing retinal arteries. Gene Expr Patterns 5:123-127. doi:10.1016/j.modgep.2004.05.004

10. Herzog Y et al (2001) Differential expression of neuropilin-1 and neuropilin-2 in arteries and veins. Mech Dev 109:115-119. doi:10.1016/S0925-4773(01)00518-4

11. Lee $P$ et al (2002) Neuropilin-1 is required for vascular development and is a mediator of VEGF-dependent angiogenesis in zebrafish. Proc Natl Acad Sci USA 99:10470-10475. doi:10.1073/ pnas. 162366299

12. Martyn U, Schulte-Merker S (2004) Zebrafish neuropilins are differentially expressed and interact with vascular endothelial growth factor during embryonic vascular development. Dev Dyn 231:33-42. doi:10.1002/dvdy.20048

13. Moyon D et al (2001) Plasticity of endothelial cells during arterial-venous differentiation in the avian embryo. Development 128:3359-3370

14. Yuan L et al (2002) Abnormal lymphatic vessel development in neuropilin 2 mutant mice. Development 129:4797-4806

15. Kaipainen A et al (1995) Expression of the fms-like tyrosine kinase 4 gene becomes restricted to lymphatic endothelium during development. Proc Natl Acad Sci USA 92:3566-3570. doi:10.1073/pnas.92.8.3566

16. Tammela $\mathrm{T}$ et al (2008) Blocking VEGFR-3 suppresses angiogenic sprouting and vascular network formation. Nature 454:656660. doi:10.1038/nature 07083

17. Lawson ND et al (2001) Notch signaling is required for arterialvenous differentiation during embryonic vascular development. Development 128:3675-3683

18. You LR et al (2005) Suppression of Notch signalling by the COUP-TFII transcription factor regulates vein identity. Nature 435:98-104. doi:10.1038/nature03511

19. Saint-Geniez M et al (2003) The msr/apj gene encoding the apelin receptor is an early and specific marker of the venous phenotype in the retinal vasculature. Gene Expr Patterns 3:467472. doi:10.1016/S1567-133X(03)00062-0

20. Dekker RJ et al (2002) Prolonged fluid shear stress induces a distinct set of endothelial cell genes, most specifically lung Kruppel-like factor (KLF2). Blood 100:1689-1698. doi:10.1182/ blood-2002-01-0046

21. Parmar KM et al (2006) Integration of flow-dependent endothelial phenotypes by Kruppel-like factor 2. J Clin Invest 116: 49-58. doi:10.1172/JCI24787

22. Huddleson JP et al (2004) Fluid shear stress induces endothelial KLF2 gene expression through a defined promoter region. Biol Chem 385:723-729. doi:10.1515/BC.2004.088

23. Benjamin LE et al (1998) A plasticity window for blood vessel remodelling is defined by pericyte coverage of the preformed endothelial network and is regulated by PDGF-B and VEGF. Development 125:1591-1598

24. le Noble F et al (2005) Control of arterial branching morphogenesis in embryogenesis: go with the flow. Cardiovasc Res 65:619-628. doi:10.1016/j.cardiores.2004.09.018

25. le Noble F et al (2004) Flow regulates arterial-venous differentiation in the chick embryo yolk sac. Development 131: 361-375. doi:10.1242/dev.00929

26. Armulik A et al (2005) Endothelial/pericyte interactions. Circ Res 97:512-523. doi:10.1161/01.RES.0000182903.16652.d7

27. Adams RH, Alitalo K (2007) Molecular regulation of angiogenesis and lymphangiogenesis. Nat Rev Mol Cell Biol 8: 464-478. doi:10.1038/nrm2183

28. Bergers G, Song S (2005) The role of pericytes in blood-vessel formation and maintenance. Neuro-oncol 7:452-464. doi:10.1215/S115 2851705000232
29. Houtchens GR et al (2008) Combined effects of microtopography and cyclic strain on vascular smooth muscle cell orientation. J Biomech 41:762-769. doi:10.1016/j.jbiomech.2007.11.027

30. Kurpinski K et al (2006) Regulation of vascular smooth muscle cells and mesenchymal stem cells by mechanical strain. Mol Cell Biomech 3:21-34

31. Liu SQ (1998) Influence of tensile strain on smooth muscle cell orientation in rat blood vessels. J Biomech Eng 120:313-320. doi:10.1115/1.2797996

32. Abraham $\mathrm{S}$ et al (2008) Integrin betal subunit controls mural cell adhesion, spreading, and blood vessel wall stability. Circ Res 102:562-570. doi:10.1161/CIRCRESAHA.107.167908

33. van Eys GJ et al (2007) Smoothelin in vascular smooth muscle cells. Trends Cardiovasc Med 17:26-30. doi:10.1016/j.tcm.2006. 11.001

34. Zanetti M et al (2004) EMILIN-1 deficiency induces elastogenesis and vascular cell defects. Mol Cell Biol 24:638-650. doi:10.1128/MCB.24.2.638-650.2004

35. Patel A et al (2006) Elastin biosynthesis: The missing link in tissue-engineered blood vessels. Cardiovasc Res 71:40-49. doi: 10.1016/j.cardiores.2006.02.021

36. Karnik SK et al (2003) A critical role for elastin signaling in vascular morphogenesis and disease. Development 130:411-423. doi: $10.1242 /$ dev.00223

37. Hellstrom M et al (2001) Lack of pericytes leads to endothelial hyperplasia and abnormal vascular morphogenesis. J Cell Biol 153:543-553. doi:10.1083/jcb.153.3.543

38. Urness LD et al (2000) Arteriovenous malformations in mice lacking activin receptor-like kinase-1. Nat Genet 26:328-331. doi:10.1038/81634

39. Li DY et al (1999) Defective angiogenesis in mice lacking endoglin. Science 284:1534-1537. doi:10.1126/science.284.5419. 1534

40. Arthur HM et al (2000) Endoglin, an ancillary TGFbeta receptor, is required for extraembryonic angiogenesis and plays a key role in heart development. Dev Biol 217:42-53. doi:10.1006/ dbio.1999.9534

41. Oh SP et al (2000) Activin receptor-like kinase 1 modulates transforming growth factor-beta 1 signaling in the regulation of angiogenesis. Proc Natl Acad Sci USA 97:2626-2631. doi:10.1073/ pnas.97.6.2626

42. Dickson MC et al (1995) Defective haematopoiesis and vasculogenesis in transforming growth factor-beta 1 knock out mice. Development 121:1845-1854

43. Davis S et al (1996) Isolation of angiopoietin-1, a ligand for the TIE2 receptor, by secretion-trap expression cloning. Cell 87: 1161-1169. doi:10.1016/S0092-8674(00)81812-7

44. Sato TN et al (1995) Distinct roles of the receptor tyrosine kinases Tie-1 and Tie-2 in blood vessel formation. Nature 376: 70-74. doi:10.1038/376070a0

45. Suri C et al (1996) Requisite role of angiopoietin-1, a ligand for the TIE2 receptor, during embryonic angiogenesis. Cell 87: 1171-1180. doi:10.1016/S0092-8674(00)81813-9

46. Foo SS et al (2006) Ephrin-B2 controls cell motility and adhesion during blood-vessel-wall assembly. Cell 124:161-173. doi: 10.1016/j.cell.2005.10.034

47. Korff T et al (2008) Role of ephrinB2 expression in endothelial cells during arteriogenesis: impact on smooth muscle cell migration and monocyte recruitment. Blood 112:73-81. doi:10.1182/blood-200712-128835

48. Wu J et al (2008) KLF2 transcription factor modulates blood vessel maturation through smooth muscle cell migration. J Biol Chem 283:3942-3950. doi:10.1074/jbc.M707882200

49. Olsson AK et al (2006) VEGF receptor signalling - in control of vascular function. Nat Rev Mol Cell Biol 7:359-371. doi:10.1038/ nrm1911 
50. Shibuya M, Claesson-Welsh L (2006) Signal transduction by VEGF receptors in regulation of angiogenesis and lymphangiogenesis. Exp Cell Res 312:549-560. doi:10.1016/j.yexcr. 2005.11.012

51. Lawson ND et al (2002) Sonic hedgehog and vascular endothelial growth factor act upstream of the Notch pathway during arterial endothelial differentiation. Dev Cell 3:127-136. doi: 10.1016/S1534-5807(02)00198-3

52. Hong CC et al (2006) Artery/vein specification is governed by opposing phosphatidylinositol-3 kinase and MAP kinase/ERK signaling. Curr Biol 16:1366-1372. doi:10.1016/j.cub.2006.05. 046

53. Jia H et al (2004) Vascular endothelial growth factor (VEGF)-D and VEGF-A differentially regulate KDR-mediated signaling and biological function in vascular endothelial cells. J Biol Chem 279:36148-36157. doi:10.1074/jbc.M401538200

54. Makinen $\mathrm{T}$ et al (2001) Isolated lymphatic endothelial cells transduce growth, survival and migratory signals via the VEGFC/D receptor VEGFR-3. EMBO J 20:4762-4773. doi:10.1093/ emboj/20.17.4762

55. Lawson ND et al (2003) Phospholipase C gamma-1 is required downstream of vascular endothelial growth factor during arterial development. Genes Dev 17:1346-1351. doi:10.1101/gad.1072203

56. Covassin LD et al (2006) Distinct genetic interactions between multiple Vegf receptors are required for development of different blood vessel types in zebrafish. Proc Natl Acad Sci USA 103: 6554-6559. doi:10.1073/pnas.0506886103

57. Takahashi $\mathrm{T}$ et al (2001) A single autophosphorylation site on KDR/Flk-1 is essential for VEGF-A-dependent activation of PLC-gamma and DNA synthesis in vascular endothelial cells. EMBO J 20:2768-2778. doi:10.1093/emboj/20.11.2768

58. Kawasaki T et al (1999) A requirement for neuropilin-1 in embryonic vessel formation. Development 126:4895-4902

59. Mukouyama YS et al (2005) Peripheral nerve-derived VEGF promotes arterial differentiation via neuropilin 1-mediated positive feedback. Development 132:941-952. doi:10.1242/dev.01675

60. Chen $\mathrm{H}$ et al (2000) Neuropilin-2 regulates the development of selective cranial and sensory nerves and hippocampal mossy fiber projections. Neuron 25:43-56. doi:10.1016/S0896-6273(00)80 870-3

61. Giger RJ et al (2000) Neuropilin-2 is required in vivo for selective axon guidance responses to secreted semaphorins. Neuron 25:29-41. doi:10.1016/S0896-6273(00)80869-7

62. Favier B et al (2006) Neuropilin-2 interacts with VEGFR-2 and VEGFR-3 and promotes human endothelial cell survival and migration. Blood 108:1243-1250. doi:10.1182/blood-2005-114447

63. Karpanen $T$ et al (2006) Functional interaction of VEGF-C and VEGF-D with neuropilin receptors. FASEB J 20:1462-1472. doi:10.1096/fj.05-5646com

64. Caunt $\mathrm{M}$ et al (2008) Blocking neuropilin-2 function inhibits tumor cell metastasis. Cancer Cell 13:331-342. doi:10.1016/j.ccr. 2008.01.029

65. Lai EC (2004) Notch signaling: control of cell communication and cell fate. Development 131:965-973. doi:10.1242/dev.01074

66. Roca C, Adams RH (2007) Regulation of vascular morphogenesis by Notch signaling. Genes Dev 21:2511-2524. doi:10.1101/ gad. 1589207

67. Krebs LT et al (2004) Haploinsufficient lethality and formation of arteriovenous malformations in Notch pathway mutants. Genes Dev 18:2469-2473. doi:10.1101/gad.1239204

68. Krebs LT et al (2000) Notch signaling is essential for vascular morphogenesis in mice. Genes Dev 14:1343-1352

69. Duarte A et al (2004) Dosage-sensitive requirement for mouse Dll4 in artery development. Genes Dev 18:2474-2478. doi: $10.1101 / \mathrm{gad} .1239004$
70. Gale NW et al (2004) Haploinsufficiency of delta-like 4 ligand results in embryonic lethality due to major defects in arterial and vascular development. Proc Natl Acad Sci USA 101:1594915954. doi:10.1073/pnas.0407290101

71. Hellstrom M et al (2007) Dll4 signalling through Notch1 regulates formation of tip cells during angiogenesis. Nature 445: 776-780. doi:10.1038/nature05571

72. Siekmann AF et al (2008) Modulation of VEGF signalling output by the Notch pathway. Bioessays 30:303-313. doi:10.1002/bies. 20736

73. Leslie JD et al (2007) Endothelial signalling by the Notch ligand Delta-like 4 restricts angiogenesis. Development 134:839-844. doi:10.1242/dev.003244

74. Suchting S et al (2007) The Notch ligand Delta-like 4 negatively regulates endothelial tip cell formation and vessel branching. Proc Natl Acad Sci USA 104:3225-3230. doi:10.1073/pnas. 0611177104

75. Lobov IB et al (2007) Delta-like ligand 4 (D114) is induced by VEGF as a negative regulator of angiogenic sprouting. Proc Natl Acad Sci USA 104:3219-3224. doi:10.1073/pnas.0611206104

76. Liu ZJ et al (2003) Regulation of Notch1 and Dll4 by vascular endothelial growth factor in arterial endothelial cells: implications for modulating arteriogenesis and angiogenesis. Mol Cell Biol 23:14-25. doi:10.1128/MCB.23.1.14-25.2003

77. Seo $S$ et al (2006) The forkhead transcription factors, Foxc1 and Foxc2, are required for arterial specification and lymphatic sprouting during vascular development. Dev Biol 294:458-470. doi:10.1016/j.ydbio.2006.03.035

78. Hayashi H, Kume T (2008) Foxc transcription factors directly regulate D114 and Hey2 expression by interacting with the VEGF-Notch signaling pathways in endothelial cells. PLoS One 3:e2401. doi:10.1371/journal.pone.0002401

79. Ioannidou $\mathrm{S}$ et al (2006) An in vitro assay reveals a role for the diaphragm protein PV-1 in endothelial fenestra morphogenesis. Proc Natl Acad Sci USA 103:16770-16775. doi:10.1073/pnas. 0603501103

80. Stan RV et al (2004) PV1 is a key structural component for the formation of the stomatal and fenestral diaphragms. Mol Biol Cell 15:3615-3630. doi:10.1091/mbc.E03-08-0593

81. Stan RV et al (1999) PV-1 is a component of the fenestral and stomatal diaphragms in fenestrated endothelia. Proc Natl Acad Sci USA 96:13203-13207. doi:10.1073/pnas.96.23.13203

82. Hnasko R et al (2002) Distribution and characterization of plasmalemma vesicle protein-1 in rat endocrine glands. J Endocrinol 175:649-661. doi:10.1677/joe.0.1750649

83. Roberts WG, Palade GE (1995) Increased microvascular permeability and endothelial fenestration induced by vascular endothelial growth factor. J Cell Sci 108(Pt 6):2369-2379

84. Esser S et al (1998) Vascular endothelial growth factor induces endothelial fenestrations in vitro. J Cell Biol 140:947-959. doi: 10.1083/jcb.140.4.947

85. Cao R et al (2004) Comparative evaluation of FGF-2-, VEGFA-, and VEGF-C-induced angiogenesis, lymphangiogenesis, vascular fenestrations, and permeability. Circ Res 94:664-670. doi:10.1161/01.RES.0000118600.91698.BB

86. LeCouter $\mathrm{J}$ et al (2001) Identification of an angiogenic mitogen selective for endocrine gland endothelium. Nature 412:877-884. doi: $10.1038 / 35091000$

87. Carpenter B et al (2005) VEGF is crucial for the hepatic vascular development required for lipoprotein uptake. Development 132:3293-3303. doi:10.1242/dev.01902

88. Lammert E et al (2003) Role of VEGF-A in vascularization of pancreatic islets. Curr Biol 13:1070-1074. doi:10.1016/S09609822(03)00378-6

89. Inai $\mathrm{T}$ et al (2004) Inhibition of vascular endothelial growth factor (VEGF) signaling in cancer causes loss of endothelial 
fenestrations, regression of tumor vessels, and appearance of basement membrane ghosts. Am J Pathol 165:35-52

90. Kamba $\mathrm{T}$ et al (2006) VEGF-dependent plasticity of fenestrated capillaries in the normal adult microvasculature. Am J Physiol Heart Circ Physiol 290:H560-H576. doi:10.1152/ajpheart.001 33.2005

91. Wallow IH, Geldner PS (1980) Endothelial fenestrae in proliferative diabetic retinopathy. Invest Ophthalmol Vis Sci 19: 1176-1183

92. Schlageter KE et al (1999) Microvessel organization and structure in experimental brain tumors: microvessel populations with distinctive structural and functional properties. Microvasc Res 58:312-328. doi:10.1006/mvre.1999.2188

93. Roberts WG et al (1998) Host microvasculature influence on tumor vascular morphology and endothelial gene expression. Am J Pathol 153:1239-1248

94. Engelhardt B (2003) Development of the blood-brain barrier. Cell Tissue Res 314:119-129. doi:10.1007/s00441-003-0751-z

95. Liebner S et al (2008) Wnt/beta-catenin signaling controls development of the blood-brain barrier. J Cell Biol 183:409-417. doi: $10.1083 /$ jcb. 200806024

96. Stenman JM et al (2008) Canonical Wnt signaling regulates organ-specific assembly and differentiation of CNS vasculature. Science 322:1247-1250. doi:10.1126/science.1164594

97. Srinivasan RS et al (2007) Lineage tracing demonstrates the venous origin of the mammalian lymphatic vasculature. Genes Dev 21:2422-2432. doi:10.1101/gad.1588407
98. Hong YK et al (2002) Prox1 is a master control gene in the program specifying lymphatic endothelial cell fate. Dev Dyn 225:351-357. doi:10.1002/dvdy.10163

99. Petrova TV et al (2002) Lymphatic endothelial reprogramming of vascular endothelial cells by the Prox-1 homeobox transcription factor. EMBO J 21:4593-4599. doi:10.1093/emboj/cdf470

100. Wigle JT et al (2002) An essential role for Prox 1 in the induction of the lymphatic endothelial cell phenotype. EMBO J 21:1505-1513. doi:10.1093/emboj/21.7.1505

101. Johnson NC et al (2008) Lymphatic endothelial cell identity is reversible and its maintenance requires Prox 1 activity. Genes Dev 22:3282-3291. doi:10.1101/gad.1727208

102. Breiteneder-Geleff S et al (1999) Angiosarcomas express mixed endothelial phenotypes of blood and lymphatic capillaries: podoplanin as a specific marker for lymphatic endothelium. Am J Pathol 154:385-394

103. Hong YK et al (2004) Lymphatic reprogramming of blood vascular endothelium by Kaposi sarcoma-associated herpesvirus. Nat Genet 36:683-685. doi:10.1038/ng1383

104. Alitalo K et al (2005) Lymphangiogenesis in development and human disease. Nature 438:946-953. doi:10.1038/nature04480

105. Francois Met al (2008) Sox 18 induces development of the lymphatic vasculature in mice. Nature 456:643-647. doi:10.1038/nature07391

106. Irrthum A et al (2003) Mutations in the transcription factor gene SOX18 underlie recessive and dominant forms of hypotrichosislymphedema-telangiectasia. Am J Hum Genet 72:1470-1478. doi: $10.1086 / 375614$ 